\title{
Qualidade de vida e incapacidade funcional entre idosos cadastrados na estratégia de saúde da família
}

\author{
Quality of life and functional disability among elderly enrolled in the family \\ health strategy
}

Árlen Almeida Duarte de Sousa', Andréa Maria Eleutério de Barros Lima Martins', Marise Fagundes Silveira'1, Wagner Luiz Mineiro Coutinho², Daniel Antunes Freitas ${ }^{1}$, Eveline Lucena Vasconcelos ${ }^{3}$, Amanda Miranda Brito Araújo ${ }^{1}$, Ana Monique Gomes Brito², Raquel Conceição Ferreira ${ }^{4}$

IUniversidade Estadual de Montes Claros (Unimontes) - Montes Claros (MG), Brasil.

${ }^{2}$ Faculdades Integradas do Norte de Minas (Funorte) - Montes Claros (MG), Brasil.

3Universidade Federal de Alagoas (UFAL) - Maceió (AL), Brasil.

${ }^{4}$ Universidade Federal de Minas Gerais (UFMG) - Belo Horizonte (MG), Brasil.

DOI: https://dx.doi.org/10.7322/abcshs.v43i1.986

\section{RESUMO}

Introdução: O comprometimento da funcionalidade do idoso traz consequências prejudiciais relacionadas à família, comunidade e ao próprio sistema de saúde. Objetivo: Estimar a ocorrência da qualidade de vida (QV) ruim entre idosos, além de verificar sua associação com incapacidade funcional, características pessoais, comportamentos de saúde e condições subjetivas de saúde bucal. Métodos: Estudo epidemiológico avaliou 361 idosos com idade igual ou superior a 60 anos, cadastrados nas três Estratégias de Saúde da Família no município de Ibiaí-MG. A QV foi medida pelo WHOQOL-bref e as variáveis independentes foram características pessoais, comportamentos de saúde, condições subjetivas de saúde bucal e incapacidade funcional. Foram feitas regressões logísticas para estimativa dos modelos múltiplos e o modelo final foi ajustado, mantendo-se as variáveis associadas com $p<0,05$. Resultados: No domínio físico, constatou-se menor chance de QV ruim entre idosos que possuíam hábitos etilistas, e maior chance entre os idosos que faziam uso de medicamento, que relataram percepção ruim/péssima da aparência dos dentes e gengivas e entre aqueles com incapacidade funcional. No domínio psicológico, observou-se menor chance de QV ruim entre homens, e maior chance entre os idosos que relataram percepção da saúde bucal como ruim/péssima e aqueles com incapacidade funcional. No domínio social, obteve-se maior chance de QV ruim entre idosos que relataram percepção ruim/péssima da aparência dos dentes e gengivas e que apresentaram incapacidade funcional. Conclusão: Constatou-se alta ocorrência de QV ruim entre idosos e evidenciouse relação entre $Q V$ ruim e incapacidade funcional.

Palavras chave: qualidade de vida; atividades cotidianas; saúde bucal; saúde do idoso; atenção primária à saúde.

\begin{abstract}
Introduction: The compromise of elderly functionality has harmful consequences to family, community and the health system. Objective: To estimate the occurrence of low quality of life (QOL) among elderly, as well as to verify its association with functional disability, personal characteristics, health behaviors and subjective oral health conditions. Methods: Epidemiological study with 361 elderly individuals aged equal or higher 60 years registered in the three Family Health Units from the city of IbiaíMG. QOL was measured by the WHOQOL-bref questionnaire and the independent variables were personal characteristics, health behaviors, subjective oral health conditions and functional disability. Logistic regressions were made to estimate the multiple models and the final model was adjusted, maintaining the variables associated with $p<0.05$. Results: In the physical domain, there was a lower chance of low QOL among elderly consuming alcohol, and a higher chance among those using medication, who reported bad/terrible perception of teeth and gums appearance and among those with functional disability. In the psychological domain, there was a lower chance of low QOL among men, and a higher chance among elderly who reported bad/terrible perception of oral health and those with functional disability. In the social domain, there was a higher chance of low QOL among the elderly who reported bad/terrible perception of the teeth and gums appearance and who presented functional disability. Conclusion: There was a high occurrence of low QOL among elderly and the relation between low QOL and functional disability was evidenced.
\end{abstract}

Keywords: quality of life; activities of daily living; oral health; health of the elderly; primary health care.

Recebido em: 04/06/2017

Revisado em: 12/09/2017

Aprovado em: 24/11/2017

Autora para correspondência: Amanda Miranda Brito Araújo - Universidade Estadual de Montes Claros - Centro de Ciências Biológicas e da Saúde Avenida Dr. Ruy Braga, s/n - Vila Mauriceia - CEP: 39401-089 - Montes Claros (MG), Brasil - E-mail: amandambaraujo@gmail.com Apoio financeiro: AADS recebeu bolsa FAPEMIG

Conflito de interesses: nada a declarar. 


\section{INTRODUÇÃO}

A capacidade funcional se faz necessária em vários aspectos da vida do idoso, seja em relação a sua família, a sua comunidade e até mesmo quando se trata do próprio sistema de saúde no qual ele está inserido. O comprometimento da sua funcionalidade ocasiona dependência física, tornando o idoso mais vulnerável nas suas atividades de vida diária, diminuindo a qualidade de vida e bem-estar do indivíduo ${ }^{1,2}$.

As atividades da vida diária são todas as atividades realizadas pelas pessoas em seu dia a dia, onde se encontram os cuidados pessoais, as questões de comunicação e ambientais, e também a mobilidade funcional ${ }^{1-3}$. Atividades Básicas de Vida Diária dizem respeito às atividades que denotam o autocuidado, representadas por ações como trocar de roupa, tomar banho, se alimentar sozinho; enfim, ações que refletem sobre a vida cotidiana individual ${ }^{3}$. As Atividades Instrumentais de Vida Diária representam ações de maior complexidade que tem representatividade relacionada à vida em comunidade. A incapacidade funcional gera dificuldades e obstáculos à liberdade e independência plena para o idoso, afetando a manutenção do estado individual quando se considera o conceito atual de saúde ${ }^{2,3}$.

A capacidade funcional pode ser entendida quando o indivíduo possui uma vida autônoma e independente dentro de suas habilidades físicas e mentais. À medida que essa capacidade vai se deteriorando, o idoso necessita de auxilio de terceiros no desempenho de suas atividades. Essa pessoa assume a função de cuidador ${ }^{4}$.

Avaliar a capacidade funcional do idoso é uma estratégia importante na atenção em saúde, uma vez que permitirá a identificação dos fatores de risco e o monitoramento da evolução clínica dos problemas de saúde, como complicação ou instauração de doença crônica, probabilidade de quedas, entre outros, possibilitando, dessa forma, a preservação da autonomia e independência do indivíduo na terceira idade ${ }^{2,4}$.

A autopercepção da saúde bucal apresenta-se como importante componente da qualidade de vida entre idosos, visto que a autopercepção negativa da saúde bucal pode afetar a vida cotidiana das pessoas. Exemplo disso é que, além de poder afetar atividades diárias elementares como fala e alimentação, também o papel coletivo pode ser influenciado através das questões estéticas relacionadas à saúde bucal. Neste contexto, uma saúde bucal comprometida pode estar relacionada a uma estética bucal negativa e esta situação resultar em constrangimento para o indivíduo afetado frente ao grupo social de seu convívio ${ }^{4,5}$.

Tendo em vista a relação da idade com a morbidade, uma das consequências do aumento do número de indivíduos idosos é uma demanda crescente por serviços de saúde 5 . No Brasil, a Atenção Primária à Saúde se desenvolve a partir de um modelo centrado na Estratégia Saúde da Família (ESF); esta deve ser para todos os usuários do Sistema Único de Saúde, também para os idosos, a porta de entrada e o seu elo junto ao sistema público de saúde $^{6,7}$. Cabe às Equipes da ESF, tanto as Equipes de Saúde da Família como as Equipes de Saúde Bucal, conhecer a realidade das famílias adstritas em seus territórios, mobilizar e envolver os indivíduos idosos em grupos e propostas que façam com que eles se tornem personagens atuantes de suas próprias condições de saúde $^{8,9}$. Assim, o objetivo a ser alcançado pelos profissionais da Atenção Primária à Saúde é de um envelhecimento saudável e ativo, contribuindo para que as limitações rotineiras aos idosos não sejam impedimentos a uma boa condição de saúde ${ }^{8,9}$.

A pesquisa justifica-se pelo número crescente de idosos, resultado da queda na taxa de mortalidade e natalidade; estes estão em busca da manutenção de suas necessidades básica de vida, bem como na procura por interação com o ambiente em que vivem com o objetivo de melhorar sua qualidade de vida. Além disso, investigar a qualidade de vida de idosos e sua funcionalidade vem se tornando cada vez mais necessário, pois os resultados podem compor um conjunto de ferramentas importantes para construção de intervenções e/ou programas que venham proporcionar bem-estar a este público.

Esse estudo buscou estimar a ocorrência da qualidade de vida ruim geral e em seus domínios físico, ambiente, social e psicológico entre idosos, além de verificar sua associação com a incapacidade funcional, características pessoais, comportamentos de saúde e condições subjetivas de saúde bucal.

\section{MÉTODOS}

Estudo epidemiológico, transversal analítico conduzido em um município brasileiro de pequeno porte populacional (Ibiaí, Norte de Minas Gerais) entre idosos cadastrados na ESF com idade igual ou superior a 60 anos completos em $1^{\circ}$ de janeiro de 2010, residentes e domiciliados na zona urbana.

Foi obtida das três equipes das ESF existentes na cidade, que cobrem $100 \%$ da população do município, uma lista com os nomes e endereços dos idosos cadastrados com idade igual o superior a 60 anos. Posteriormente, dez pesquisadores, previamente treinados e calibrados por técnica de Role Play para aplicação dos instrumentos, percorreram todos os domicílios para buscar pelos idosos, sendo os dados coletados por meio de entrevistas utilizando um formulário contendo todos os instrumentos. O processo de coleta de dados foi realizado entre os anos de 2011 e 2012.

Os critérios de inclusão para participação da pesquisa foram idosos com 60 anos ou mais, que apresentassem condição cognitiva preservada confirmada a partir da aplicação do Mini Exame do Estado Mental (MEEM). Adotaram-se como critérios de exclusão idosos que apresentaram cinco ou mais patologias instaladas diagnosticadas por um médico, déficit visual e/ou auditivo não corrigido, afasia e/ou outros comprometimentos neurológicos que os impediam de responder os questionários, idosos acamados, em uso de cadeira de rodas e/ou outros dispositivos auxiliares da marcha que impossibilitassem o desempenho das atividades físicas, estar sendo assistido nos níveis secundário e ou terciário de assistência à saúde, questionários preenchidos de forma incompleta. 
Dos 479 idosos contidos na lista obtida da ESF, 449 (93,74\%) foram encontrados, $09(1,87 \%)$ mudaram para outra cidade ou para a zona rural, $04(0,84 \%)$ estavam muito debilitados ou internados sem condições de participar do estudo, 06 (1,25\%) não foram encontrados por estarem fora do município e 08 (1,67\%) por inexistência do endereço; 03 idosos $(0,63 \%)$ tinham falecido. Por outro lado, 27 idosos que não constavam na lista da ESF por falha no cadastramento foram adicionados, totalizando 476 idosos elegíveis para o estudo. Destes, a taxa de recusa foi de 3,57\% $(n=17)$ e $459(96,43 \%)$ responderam pelo menos a um dos instrumentos de coleta de dados iniciais, dentre os quais o relativo aos dados sociodemográficos; 61 (12,8\%) idosos foram excluídos por confirmação de problemas cognitivos após a aplicação do MEEM. O motivo da recusa em responder os questionários não foi explicitado pelos idosos abordados para o estudo, apenas não demonstraram interesse em participar. Entre estes, 361 idosos responderam as variáveis de interesse neste estudo e apresentaram estado cognitivo preservado (questionário WHOQOL-bref, Escala de Lawton e Brody e Escala de Katz), definindo uma perda de 21,4\%.

A variável dependente, qualidade de vida, foi avaliada utilizando o questionário WHOQOL-bref, versão validada no Brasil ${ }^{9}$. Este instrumento possui duas questões de ordem geral e 26 questões relacionadas a quatro domínios (físico, psicológico, ambiente e social). As opções de resposta seguem uma escala do tipo Likert, que mede intensidade, frequência, capacidade e avaliação. Cada uma dessas medidas vai de "nada a extremamente" (intensidade), "nunca a sempre" (frequência), "nada a completamente" (capacidade) e "muito satisfeito a muito insatisfeito e muito ruim a muito bom" (avaliação).

Os escores de cada domínio do questionário WHOQOL-bref foram transformados em escalas de 0 a 100, sendo considerada a melhor qualidade de vida aquela que conseguir o maior esco$\mathrm{re}^{10,11}$. Os escores finais de cada um dos domínios foram dicotomizados pelo Intervalo de Confiança (IC). Os idosos que apresentaram menores escores (valores inferiores ao limite inferior do IC da média) foram considerados com qualidade de vida ruim (Domínio físico: 61,62; Domínio psicológico: 64,45; Domínio ambiente: 58,73; Domínio Social: 75,66). Os respondentes que deixaram de preencher ou preencheram incorretamente seis ou mais questões do questionário WHOQOL-bref foram excluídos do estudo $(\mathrm{n}=37)$.

A incapacidade funcional foi avaliada pela escala de atividades instrumentais de vida diárias, que aborda atividades complexas com maior independência social ${ }^{1,7}$. Desenvolvida por Lawton e Brody $^{12}$ foi adaptada ao contexto brasileiro ${ }^{3}$, sendo composta por sete questões estruturadas relacionadas ao uso do telefone, viagem, compras, preparo de refeições, trabalho doméstico, uso de medicamentos e manuseio do dinheiro. Cada questão contém uma escala com três pontos, variando numericamente de 01 a 03 , sendo a soma mínima de 07 pontos e a máxima de 21 . Em relação às categorias relacionadas às atividades de vida diária, o idoso teve três possibilidades de resposta, sendo elas independência, necessidade de ajuda parcial e necessidade de ajuda total ${ }^{2}$.
Para definição da incapacidade funcional foram propostas três categorias, cujos pontos de cortes foram definidos pelos percentis: Independente (percentil 100), Dependência Parcial (percentil > 25 $\mathrm{a}<100$ ) e Dependência Total (percentil 25) ${ }^{13}$.

Com o objetivo de avaliar a incapacidade funcional para desempenhar Atividades Básicas de Vida Diária, utilizou-se a Escala de Katz criada por Sidney Katz et al. ${ }^{14}$, que analisa a capacidade para tomar banho, vestir uma roupa, usar o banheiro, mover-se da cama para a cadeira e vice-versa, comer sozinho e controlar o tônus esfincteriano. Tal escala, amplamente utilizada em pesquisas e adaptada para a cultura brasileira por Lino et al. ${ }^{15}$ é reconhecida pelo Ministério da Saúde do Brasil ${ }^{16}$ para avaliação da pessoa idosa na atenção básica ${ }^{2}$.

Para as seis atividades são propostas três alternativas de resposta; o resultado possibilita que o idoso seja direcionado a uma classificação, variando de A a Outro, a saber:

- A) independente para todas as atividades;

- B) independente para todas as atividades menos uma;

- C) independente para todas as atividades menos banho e mais uma adicional;

- D) independente para todas as atividades menos banho, vestir-se e mais uma adicional;

- E) independente para todas as atividades menos banho, vestir-se, ir ao banheiro e mais uma adicional;

- F) independente para todas as atividades menos banho, vestir-se, ir ao banheiro, transferência e mais uma adicional;

- G) dependente para todas as atividades;

- Outro) Dependente em pelo menos duas funções, mas que não se classificasse em C, D, E, e F ${ }^{16}$.

Utilizou-se o modelo proposto por Rubenstein et al. ${ }^{17}$ elaborado com a aceitação de Sidney Katz para categorização dos indivíduos: Mais Funcional (junção das situações A e B), Funcionalidade Intermediária (junção das situações C, D e E) e Menos Funcional (junção das situações F e G).

As outras variáveis independentes foram referentes às características pessoais, comportamentos de saúde e condições subjetivas de saúde bucal. As características pessoais foram reunidas em três subgrupos: Sociodemográficas (idade: 60 a 70, maior ou igual a 71; cor da pele autodeclarada: branco ou amarelo, pardo/negro; sexo: feminino, masculino; situação conjugal: com companheiro, sem companheiro; escolaridade em anos de estudo: 0, 1-4, 5 ou mais ${ }^{18}$; Disponibilidade de Recursos (renda per capita em reais: $>255,00, \leq 255,00$ ); Saúde Geral (presença de doenças crônicas: não, sim; uso de medicamentos: não, sim).

As variáveis relacionadas a comportamentos de saúde foram hábitos tabagistas (ausente, presente), hábitos etilistas (ausente, presente) e atividade física. Essa última variável foi avaliada pelo instrumento Curitibativa. O instrumento considera as atividades realizadas na última semana, segmentadas em três grupos: prática de atividades físicas sistemáticas, prática de tarefas domésticas ou de trabalho-esforço físico e atividades sociais ou de lazer ${ }^{19}$. Cada um 
destes itens foi pontuado de acordo com uma escala que variou de 2,5 a 4, multiplicado pelo número de vezes que o sujeito praticou tal atividade durante uma semana e pelo tempo gasto para a realização de tais atividades, gerando assim um escore final. Os seguintes pontos de corte definiram o nível da atividade física do idoso: de 0 a 32: inativo, de 33 a 82: pouco ativo, de 83 a 108: moderadamente ativo, de 109 a 133: ativo, e 134 ou mais: muito ativo ${ }^{20}$. Para análises múltiplas, foram criadas duas categorias: ativo (muito ativo, ativo) e inativo (inativo, pouco ativo, moderadamente ativo).

As condições individuais de saúde bucal foram: percepção da saúde bucal; percepção da aparência de dentes e gengivas; e percepção da fala devido aos dentes e gengivas (ótima/boa, regular, ruim/péssima) ${ }^{21}$.

Foi utilizado para análise de dados o programa Statistical Package for the Social Sciences (SPSS) ${ }^{\circledR}$ versão 17.0 for Windows. A estatística descritiva foi realizada com obtenção de frequências absoluta e relativa das variáveis categóricas e ordinais, enquanto a média, mediana e desvio padrão foram utilizadas para as variáveis quantitativas. $\mathrm{Na}$ análise univariada, a associação entre as variáveis independentes (Atividades Instrumentais de Vida Diária, Atividades Básicas de Vida Diária, características pessoais, comportamentos em saúde e condições subjetivas de saúde bucal) e a dependente (Domínios do questionário WHOQOL-bref) foi verificada por meio do teste qui-quadrado, com obtenção dos valores de Odds Ratio, sua significância estatística e intervalo de confiança 95\%. As variáveis associadas com a variável dependente $(\mathrm{p} \leq 0,20)$ na análise univariada foram incluídas nas análises múltiplas. Foram feitas regressões logísticas para estimativa dos modelos múltiplos. O modelo final foi ajustado, mantendo-se as variáveis associadas com $\mathrm{p}<0,05$.

O estudo foi aprovado pelo Comitê de Ética em Pesquisa da Universidade Estadual de Montes Claros (CEP/UNIMONTES, parecer consubstanciado n. 2903/11). Os pesquisadores comprometeram-se em manter o sigilo

\section{RESULTADOS}

Observou-se que a média de idade dos idosos foi de 71,24 anos ( \pm 8,53; IC95\%: 70,15-72,32), estudaram em média 1,82 anos ( \pm 2,63; IC95\%: 1,49-2,16) e possuíam renda per capita média, em reais, de R\$352,62 ( \pm 265,89; IC95\%: 318,81-386,43)- salário mínimo de referência: $\mathrm{R} \$ 622,00$. A maioria era do sexo feminino e se enquadrava na categoria negro/pardo. A caracterização dos idosos quanto às variáveis investigadas foi representada na Tabela 1.

Identificou-se uma maior ocorrência de qualidade de vida ruim no domínio social $(58,4 \% ; \mathrm{n}=211)$ e físico $(45,2 \% ; \mathrm{n}=163)$ do WHOQOL-bref, seguidos pelos domínios ambiente (43,8\%; $\mathrm{n}=158)$ e psicológico $(36,8 \% ; \mathrm{n}=133)$. A Qualidade de Vida Geral obteve ocorrência de qualidade de vida ruim de 41,3\% ( $n=149)$.

Em relação às Atividades Instrumentais de Vida Diária observou-se que $29,1 \%(n=105)$ dos idosos enquadraram-se como Dependência total, 63,2\% $(\mathrm{n}=228)$ como Dependência parcial e apenas 7,8\% (n=28) como Independência; o valor médio alcançado foi de 16,73 ( \pm 3,115; IC95\%: 16,41-17,06), variando de 7 a 21 . $\mathrm{Na}$ avaliação das Atividades Básicas de Vida Diária constatou-se

Tabela 1: Distribuição da população idosa quanto a características pessoais, comportamentos em saúde, atividade física e condições subjetivas de saúde bucal. Ibiaí, Minas Gerais, 2011-2012. n=361

\begin{tabular}{|c|c|c|}
\hline Características pessoais & $\mathbf{n}$ & $\%$ \\
\hline \multicolumn{3}{|l|}{ Idade (anos) } \\
\hline 60 a 70 & 170 & 47,1 \\
\hline 71 a 102 & 191 & 52,9 \\
\hline \multicolumn{3}{|l|}{ Sexo } \\
\hline Feminino & 213 & 59,0 \\
\hline Masculino & 148 & 41,0 \\
\hline \multicolumn{3}{|l|}{ Cor da pele autodeclarada } \\
\hline Branco/Amarelo & 56 & 15,5 \\
\hline Negro/Pardo & 305 & 84,5 \\
\hline \multicolumn{3}{|l|}{ Situação conjugal } \\
\hline Com companheiro & 186 & 51,5 \\
\hline Sem companheiro & 175 & 48,5 \\
\hline \multicolumn{3}{|l|}{ Escolaridade em anos* } \\
\hline 5 ou mais & 31 & 8,8 \\
\hline 1 a 4 & 145 & 41,3 \\
\hline 0 & 175 & 49,9 \\
\hline \multicolumn{3}{|l|}{ Renda per capita em reais* } \\
\hline$>255,00$ & 117 & 48,8 \\
\hline$<$ ou igual a 255,00 & 123 & 51,2 \\
\hline \multicolumn{3}{|l|}{ Doença crônica existente } \\
\hline Não & 40 & 11,1 \\
\hline Sim & 321 & 88,9 \\
\hline \multicolumn{3}{|l|}{ Uso de medicamento } \\
\hline Não & 88 & 24,4 \\
\hline $\operatorname{Sim}$ & 273 & 75,6 \\
\hline \multicolumn{3}{|l|}{ Comportamentos em saúde } \\
\hline \multicolumn{3}{|l|}{ Hábitos tabagistas* } \\
\hline Ausente & 180 & 51,1 \\
\hline Presente & 172 & 48,9 \\
\hline \multicolumn{3}{|l|}{ Hábitos etilistas* } \\
\hline Ausente & 198 & 56,3 \\
\hline Presente & 154 & 43,8 \\
\hline \multicolumn{3}{|l|}{ Atividade física* } \\
\hline Muito ativo & 44 & 12,2 \\
\hline Ativo & 11 & 3,1 \\
\hline Moderadamente ativo & 16 & 4,5 \\
\hline Pouco ativo & 72 & 20,1 \\
\hline Inativo & 215 & 60,1 \\
\hline \multicolumn{3}{|c|}{ Condições subjetivas de saúde bucal } \\
\hline \multicolumn{3}{|l|}{ Percepção da saúde bucal* } \\
\hline Ótima/boa & 262 & 74,6 \\
\hline Regular & 70 & 19,9 \\
\hline Ruim/péssima & 19 & 5,5 \\
\hline \multicolumn{3}{|c|}{ Percepção da aparência dentes e gengivas* } \\
\hline Ótima/boa & 256 & 73,4 \\
\hline Regular & 48 & 13,8 \\
\hline Ruim/péssima & 45 & 12,8 \\
\hline \multicolumn{3}{|c|}{ Percepção da fala devido dentes e gengivas* } \\
\hline Ótima/boa & 289 & 82,8 \\
\hline Regular & 45 & 12,9 \\
\hline Ruim/péssima & 15 & 4,3 \\
\hline
\end{tabular}

*Número de respondentes inferior ao número de participantes. 
que $98,6 \%(n=356)$ dos idosos foram categorizados como Mais Funcional 0,6\% (n=02) como Funcionalidade Intermediária e como Menos Funcional 0,8\% ( $=03$ ) (Tabela 2).

$\mathrm{Na}$ análise univariada, constatou-se que variáveis pertencentes Atividades Instrumentais de Vida Diária, atividade física, características pessoais, comportamentos em saúde e condições subjetivas de saúde bucal estiveram estatisticamente associadas $(\mathrm{p} \leq 0,05)$ aos domínios da qualidade de vida dos idosos (Tabela 3 e Tabela 4).

No domínio físico, constatou-se menor chance de qualidade de vida ruim entre idosos que possuíam hábitos etilistas, e maior chance entre aqueles que faziam uso de medicamento, que relataram percepção ruim/péssima da aparência dos dentes e gengivas e entre aqueles com incapacidade funcional. No domínio psicológico, foi observada menor chance de qualidade de vida ruim entre os homens, maior chance entre os idosos que relataram percepção da saúde bucal como ruim/péssima e aqueles com incapacidade funcional. No domínio social, houve maior chance de qualidade de vida ruim entre os idosos que relataram percepção ruim/péssima da aparência dos dentes e gengivas e que apresentaram incapacidade funcional (Tabela 5). No domínio ambiente, nenhuma das variáveis analisadas apresentou associação estatística significante com qualidade de vida.

\section{DISCUSSÃO}

A funcionalidade é um aspecto fundamental na análise do processo saúde doença dos idosos, devendo ser considerada na avaliação da qualidade de vida nesse grupo etário ${ }^{22}$. As Atividades Instrumentais de Vida Diária são representadas por ações de maior complexidade do que as Atividades Básicas de Vida Diária; sendo que estas primeiras apresentam maior relação com o convívio social do que com as condições individuais. Neste sentido, espera-se que as Atividades Básicas de Vida Diária não sejam as primeiras atividades diárias a serem comprometidas no cotidiano dos idosos. No presente estudo, houve associação entre capacidade funcional e qualidade de vida nos domínios físico, psicológico e social nos idosos de Ibiaí. No tocante à qualidade de vida, os prejuízos para Atividades Instrumentais de Vida Diária e Atividades Básicas de Vida Diária podem representar perda de qualidade de vida para idosos ${ }^{23,24}$.

Segundo a OMS, qualidade de vida é a percepção que um indivíduo tem sobre sua situação de vida dentro do contexto da cultura e sistema de valores no qual está inserido, estando esta posição de acordo com suas metas, expectativas e preocupações, de forma subjetiva e multidimensional ${ }^{11}$.

No presente estudo, foi observada maior chance de qualidade de vida ruim no domínio físico entre os idosos que apresentaram dependência total para as Atividades Instrumentais de Vida Diária. O domínio físico da qualidade de vida é um construto que incorpora a percepção dos indivíduos quanto a aspectos da saúde física e limitações decorrentes do seu comprometimento (capacidade de locomoção, energia para o dia a dia, incapacidade devido a dor, uso de medicamentos, qualidade do sono ${ }^{25}$. Apresentar uma incapacidade funcional nas atividades instrumentais aumentou a chance do indivíduo perceber sua qualidade de vida como ruim. Idosos com dependência para compras, preparo de refeições, trabalho doméstico, viagens e uso de telefones podem identificar essas limitações e manifestá-las por meio da percepção ruim ou insatisfação com aspectos da qualidade de vida no domínio físico.

Houve maior chance de qualidade de vida ruim no domínio psicológico naqueles idosos dependentes totais para as Atividades Instrumentais de Vida Diária. A qualidade de vida em seu domínio psicológico diz respeito à percepção do indivíduo quanto ao seu desempenho coletivo e a influência desses sobre como ele se enxerga em sua própria vivência. Nesse contexto, as dificuldades para realização das atividades cotidianas, como uso do telefone, manuseio do dinheiro e preparo de refeições comprometem a autonomia do idoso e seu sentido de utilidade na vida. A qualidade de vida ruim

Tabela 2: Descrição do grau de incapacidade funcional para as Atividades Instrumentais de Vida Diária e Atividades Básicas de Vida Diária em idosos. Ibiaí, Minas Gerais, 2011-2012

\begin{tabular}{|c|c|c|c|c|c|c|}
\hline & \multicolumn{2}{|c|}{ Independente } & \multicolumn{2}{|c|}{$\begin{array}{c}\text { Necessita de } \\
\text { assistência/ajuda }\end{array}$} & \multicolumn{2}{|c|}{$\begin{array}{c}\text { Necessita de ajuda tota } \\
\text { ou é incapaz }\end{array}$} \\
\hline & $\mathbf{n}$ & $\%$ & $\mathbf{n}$ & $\%$ & $\mathbf{n}$ & $\%$ \\
\hline \multicolumn{7}{|l|}{ Atividades Instrumentais } \\
\hline Uso do telefone* & 179 & 49,7 & 90 & 25,0 & 91 & 25,3 \\
\hline Viagens* $^{*}$ & 106 & 29,8 & 181 & 50,8 & 69 & 19,4 \\
\hline Realização de compras* & 216 & 60,5 & 79 & 22,1 & 62 & 17,4 \\
\hline Preparo das refeições* & 269 & 74,7 & 28 & 7,8 & 63 & 17,5 \\
\hline Tarefas domésticas* & 140 & 38,9 & 141 & 39,2 & 79 & 21,9 \\
\hline Uso de medicamentos* & 282 & 78,3 & 63 & 17,5 & 15 & 4,2 \\
\hline Manuseio do dinheiro* & 251 & 69,8 & 52 & 14,4 & 57 & 15,8 \\
\hline \multicolumn{7}{|l|}{ Atividades Básicas } \\
\hline Banhar-se* & 353 & 98,1 & 02 & 0,6 & 05 & 1,4 \\
\hline Vestir-se & 355 & 98,3 & 03 & 0,8 & 03 & 0,8 \\
\hline Ir ao banheiro & 359 & 99,4 & 01 & 0,3 & 01 & 0,3 \\
\hline Transferência & 358 & 99,2 & 02 & 0,6 & 01 & 0,3 \\
\hline Urinar e/ou evacuar* & 339 & 93,9 & 20 & 5,5 & 01 & 0,3 \\
\hline Alimentar-se & 359 & 99,4 & 01 & 0,3 & 01 & 0,3 \\
\hline
\end{tabular}

*Número de respondentes inferior ao número de participantes. 
Tabela 3: Análise univariada entre os domínios físico e psicológico da qualidade de vida ruim e as variáveis independentes. Ibiaí, Minas Gerais, 2011-2012. $n=361$

\begin{tabular}{|c|c|c|c|c|c|c|}
\hline \multirow{2}{*}{ Variáveis } & \multicolumn{3}{|c|}{ Domínio Físico } & \multicolumn{3}{|c|}{ Domínio Psicológico } \\
\hline & OR & IC (95\%) & Valor $\mathrm{p}^{\dagger}$ & OR & IC (95\%) & Valor $\mathrm{p}^{\dagger}$ \\
\hline \multicolumn{7}{|l|}{ Características pessoais } \\
\hline \multicolumn{7}{|l|}{ Idade (anos) } \\
\hline 60 a 70 & 1 & & & 1 & & \\
\hline 71 a 102 & 1,24 & 0,$82 ; 1,88$ & 0,313 & 0,98 & 0,$64 ; 1,51$ & 0,936 \\
\hline \multicolumn{7}{|l|}{ Sexo } \\
\hline Feminino & 1 & & & 1 & & \\
\hline Masculino & 0,63 & 0,$41 ; 0,97$ & $0,035^{*}$ & 0,50 & 0,$32 ; 0,79$ & $0,003^{*}$ \\
\hline \multicolumn{7}{|l|}{ Cor da pele autodeclarada } \\
\hline Branco/Amarelo & 1 & & & 1 & & \\
\hline Negro/Pardo & 1,59 & 0,$88 ; 2,87$ & $0,123^{*}$ & 0,81 & 0,$45 ; 1,45$ & 0,475 \\
\hline \multicolumn{7}{|l|}{ Situação conjugal } \\
\hline Com companheiro & 1 & & & 1 & & \\
\hline Sem companheiro & 1,37 & 0,$90 ; 2,07$ & $0,139^{*}$ & 1,50 & 0,$98 ; 2,31$ & $0,063^{*}$ \\
\hline \multicolumn{7}{|l|}{ Escolaridade em anos } \\
\hline 5 ou mais & 1 & & & 1 & & \\
\hline 1 a 4 & 0,95 & 0,$43 ; 2,09$ & 0,898 & 1,14 & 0,$48 ; 2,66$ & 0,769 \\
\hline 0 & 1,37 & 0,$63 ; 2,96$ & 0,426 & 1,79 & 0,$78 ; 4,11$ & $0,170^{*}$ \\
\hline \multicolumn{7}{|l|}{ Renda per capita em reais } \\
\hline $\mathrm{R} \$ 255,00$ ou mais & 1 & & & 1 & & \\
\hline Menor do que $\mathrm{R} \$ 255,00$ & 1,19 & 0,$72 ; 1,99$ & 0,487 & 1,03 & 0,$61 ; 1,74$ & 0,912 \\
\hline \multicolumn{7}{|l|}{ Doença crônica } \\
\hline Não & 1 & & & 1 & & \\
\hline Sim & 2,07 & 1,$02 ; 4,22$ & $0,041^{*}$ & 1,24 & 0,$62 ; 2,49$ & 0,546 \\
\hline \multicolumn{7}{|l|}{ Uso de medicamento } \\
\hline Não & 1 & & & 1 & & \\
\hline Sim & 2,24 & 1,$34 ; 3,74$ & $0,002^{*}$ & 1,54 & 0,$91 ; 2,58$ & $0,103^{*}$ \\
\hline \multicolumn{7}{|l|}{ Comportamentos em saúde } \\
\hline \multicolumn{7}{|l|}{ Hábitos tabagistas } \\
\hline Ausente & 1 & & & 1 & & \\
\hline Presente & 0,97 & 0,$64 ; 1,47$ & 0,882 & 0,84 & 0,$55 ; 1,29$ & 0,436 \\
\hline \multicolumn{7}{|l|}{ Hábitos etilistas } \\
\hline Ausente & 1 & & & 1 & & \\
\hline Presente & 0,64 & 0,$42 ; 0,98$ & $0,039^{*}$ & 0,78 & 0,$51 ; 1,22$ & 0,278 \\
\hline \multicolumn{7}{|l|}{ Atividade física } \\
\hline Ativo & 1 & & & 1 & & \\
\hline Inativo & 1,89 & 1,$03 ; 3,46$ & $0,038^{*}$ & 2,12 & 1,$09 ; 4,11$ & $0,024^{*}$ \\
\hline \multicolumn{7}{|c|}{ Condições subjetivas de saúde bucal } \\
\hline \multicolumn{7}{|c|}{ Percepção da saúde bucal } \\
\hline Ótima/boa & 1 & & & 1 & & \\
\hline Regular & 1,44 & 0,$85 ; 2,44$ & $0,179^{*}$ & 1,66 & 0,$97 ; 284$ & $0,062^{*}$ \\
\hline Ruim/péssima & 1,87 & 0,$73 ; 4,80$ & $0,193^{*}$ & 2,19 & 0,$86 ; 5,60$ & $0,099^{*}$ \\
\hline Percepção da aparência den & & & & & & \\
\hline Ótima/boa & 1 & & & 1 & & \\
\hline Regular & 0,79 & 0,$42 ; 1,50$ & 0,482 & 1,39 & 0,$739 ; 2,604$ & 0,308 \\
\hline Ruim/péssima & 2,65 & 1,$36 ; 5,17$ & $0,004^{*}$ & 2,03 & 1,$072 ; 3,848$ & $0,030^{*}$ \\
\hline Percepção da fala devido den & & & & & & \\
\hline Ótima/boa & 1 & & & 1 & & \\
\hline Regular & 0,99 & 0,$53 ; 1,87$ & 0,981 & 1,40 & 0,$74 ; 2,64$ & 0,297 \\
\hline Ruim/péssima & 1,42 & 0,$50 ; 4,01$ & 0,511 & 0,64 & 0,$19 ; 2,05$ & 0,450 \\
\hline Incapacidade funcional & & & & & & \\
\hline Atividades instrumentais de & & & & & & \\
\hline Independência & 1 & & & 1 & & \\
\hline Dependência parcial & 1,11 & 0,$49 ; 2,51$ & 0,801 & 2,60 & 0,$87 ; 7,79$ & $0,087^{*}$ \\
\hline Dependência total & 3,05 & 1,$28 ; 7,26$ & $0,012^{*}$ & 8,00 & 2,$59 ; 24,69$ & $0,000^{*}$ \\
\hline
\end{tabular}

\footnotetext{
${ }^{*}$ Considerou-se estatisticamente significante valor com $\mathrm{p} \leq 0,20$. ${ }^{\top}$ Teste qui-quadrado.
} 
Tabela 4: Análise univariada entre os domínios ambiente e social da qualidade de vida ruim e as variáveis independentes. Ibiaí, Minas Gerais, 2011-2012. $n=361$

\begin{tabular}{|c|c|c|c|c|c|c|}
\hline \multirow{2}{*}{ Variáveis } & \multicolumn{3}{|c|}{ Domínio Ambiente } & \multicolumn{3}{|c|}{ Domínio Social } \\
\hline & OR & IC (95\%) & Valor $\mathrm{p}^{\dagger}$ & OR & IC (95\%) & Valor $\mathrm{p}^{\dagger}$ \\
\hline \multicolumn{7}{|l|}{ Características pessoais } \\
\hline \multicolumn{7}{|l|}{ Idade (anos) } \\
\hline 60 a 70 & 1 & & & 1 & & \\
\hline 71 a 102 & 0,81 & 0,$54 ; 1,23$ & 0,329 & 0,77 & 0,$51 ; 1,18$ & 0,228 \\
\hline \multicolumn{7}{|l|}{ Sexo } \\
\hline Feminino & 1 & & & 1 & & \\
\hline Masculino & 0,60 & 0,$39 ; 0,92$ & $0,020^{*}$ & 0,77 & 0,$50 ; 1,18$ & 0,232 \\
\hline \multicolumn{7}{|l|}{ Cor da pele autodeclarada } \\
\hline Branco/Amarelo & 1 & & & 1 & & \\
\hline Negro/Pardo & 0,88 & 0,$49 ; 1,56$ & 0,662 & 0,89 & 0,$50 ; 1,60$ & 0,708 \\
\hline \multicolumn{7}{|l|}{ Situação conjugal } \\
\hline Com companheiro & 1 & & & 1 & & \\
\hline Sem companheiro & 1,33 & 0,$88 ; 2,03$ & $0,174^{*}$ & 0,94 & 0,$62 ; 1,43$ & 0,784 \\
\hline \multicolumn{7}{|l|}{ Escolaridade em anos } \\
\hline 5 ou mais & 1 & & & 1 & & \\
\hline 1 a 4 & 0,81 & 0,$37 ; 1,77$ & 0,596 & 0,44 & 0,$18 ; 1,05$ & $0,064^{*}$ \\
\hline 0 & 1,02 & 0,$48 ; 2,20$ & 0,955 & 0,49 & 0,$21 ; 1,15$ & $0,100^{*}$ \\
\hline \multicolumn{7}{|l|}{ Renda per capita em reais } \\
\hline $\mathrm{R} \$ 255,00$ ou mais & 1 & & & 1 & & \\
\hline Menor do que $\mathrm{R} \$ 255,00$ & 1,13 & 0,$67 ; 1,88$ & 0,647 & 0,95 & 0,$57 ; 1,59$ & 0,839 \\
\hline \multicolumn{7}{|l|}{ Doença crônica } \\
\hline Não & 1 & & & 1 & & \\
\hline Sim & 0,84 & 0,$44 ; 1,63$ & 0,614 & 1,31 & 0,$68 ; 2,54$ & 0,418 \\
\hline \multicolumn{7}{|l|}{ Uso de medicamento } \\
\hline Não & 1 & & & 1 & & \\
\hline Sim & 0,72 & 0,$44 ; 1,16$ & $0,175^{*}$ & 1,09 & 0,$67 ; 1,78$ & 0,721 \\
\hline \multicolumn{7}{|l|}{ Comportamentos em saúde } \\
\hline \multicolumn{7}{|l|}{ Hábitos tabagistas } \\
\hline Ausente & 1 & & & 1 & & \\
\hline Presente & 0,84 & 0,$55 ; 1,28$ & 0,418 & 0,90 & 0,$59 ; 1,38$ & 0,642 \\
\hline \multicolumn{7}{|l|}{ Hábitos etilistas } \\
\hline Ausente & 1 & & & 1 & & \\
\hline Presente & 0,69 & 0,$45 ; 1,05$ & $0,085^{*}$ & 1,07 & 0,$69 ; 1,64$ & 0,754 \\
\hline \multicolumn{7}{|l|}{ Atividade física } \\
\hline Ativo & 1 & & & 1 & & \\
\hline Inativo & 1,44 & 0,$79 ; 2,61$ & 0,224 & 1,01 & 0,$56 ; 1,81$ & 0,974 \\
\hline \multicolumn{7}{|c|}{ Condições subjetivas de saúde bucal } \\
\hline \multicolumn{7}{|c|}{ Percepção da saúde bucal } \\
\hline Ótima/boa & 1 & & & 1 & & \\
\hline Regular & 1,21 & 0,$71 ; 2,06$ & 0,474 & 1,70 & 0,$98 ; 2,96$ & $0,061^{*}$ \\
\hline Ruim/péssima & 1,22 & 0,$48 ; 3,11$ & 0,671 & 4,44 & 1,$26 ; 15,59$ & $0,020^{*}$ \\
\hline Percepção da aparência dent & & & & & & \\
\hline Ótima/boa & 1 & & & 1 & & \\
\hline Regular & 0,95 & 0,$51 ; 1,77$ & 0,867 & 1,26 & 0,$67 ; 2,37$ & 0,464 \\
\hline Ruim/péssima & 1,39 & 0,$74 ; 2,62$ & 0,321 & 2,90 & 1,$38 ; 6,11$ & $0,005^{*}$ \\
\hline Percepção da fala devido der & & & & & & \\
\hline Ótima/boa & 1 & & & 1 & & \\
\hline Regular & 1,10 & 0,$59 ; 1,07$ & 0,766 & 1,59 & 0,$81 ; 3,13$ & $0,174^{*}$ \\
\hline Ruim/péssima & 0,46 & 0,$14 ; 1,47$ & $0,189^{*}$ & 0,48 & 0,$17 ; 1,38$ & $0,175^{\star}$ \\
\hline Incapacidade funcional & & & & & & \\
\hline Atividades instrumentais de $v$ & & & & & & \\
\hline Independência & 1 & & & 1 & & \\
\hline Dependência parcial & 0,94 & 0,$42 ; 2,09$ & 0,872 & 2,37 & 1,$06 ; 5,29$ & $0,035^{\star}$ \\
\hline Dependência total & 2,14 & 0,$91 ; 5,02$ & $0,080^{*}$ & 2,23 & 0,$95 ; 5,23$ & $0,065^{\star}$ \\
\hline
\end{tabular}

${ }^{*}$ Considerou-se estatisticamente significante valor com $\mathrm{p} \leq 0,20$. ${ }^{\top}$ Teste qui-quadrado. 
manifestada nesse domínio demonstra que a capacidade funcional pode ter consequências para os sentimentos e percepção da vida pelos idosos, podendo agravar e ou desencadear um quadro de tristeza, depressão, de insatisfação consigo mesmo e com a vida ${ }^{2,26,27}$.

O envelhecimento populacional traz consigo situações que necessitam ser compreendidas, para que as ações de saúde e cuidado possam minimizar as incapacidades e limitações associadas à velhice. Um dos casos mais frequentes é que os idosos tendem a ficar sozinhos pelo fenômeno natural de envelhecimento dos filhos; em alguns casos as casas possuem somente idosos como moradores $^{27,28}$. A capacidade funcional dos idosos permitirá uma maior desenvoltura no contexto das suas necessidades e possibilitará que eles se desenvolvam positivamente nas suas relações sociais.
Atingir a longevidade é, sem dúvidas, uma das maiores conquistas da segunda metade do século XX. A expectativa de vida vem aumentando visivelmente e trata-se, nos dias de hoje, de uma conquista social. No entanto, preconiza-se que esses anos sejam vividos com certa qualidade ${ }^{28}$. O envelhecimento populacional exigirá uma maior demanda tanto sanitária e ambiental, quanto social e econômica. Dessa forma, faz-se necessária a adoção de políticas específicas que busquem propiciar um envelhecimento ativo e saudável, no qual a autonomia e, principalmente, a dignidade do idoso devam ser sempre respeitadas ${ }^{29}$.

No domínio social, os idosos com dependência total apresentaram maior chance de qualidade de vida ruim comparados aos independentes. Por outro lado, neste domínio não houve diferença

Tabela 5: Modelo final dos fatores associados aos domínios físico, psicológico e social da qualidade de vida ruim. Ibiaí, Minas Gerais, 2011-2012. $n=361$

\begin{tabular}{|c|c|c|c|c|c|c|}
\hline \multirow{2}{*}{ Variáveis } & \multicolumn{3}{|c|}{ Domínio Físico } & \multicolumn{3}{|c|}{ Domínio Psicológico } \\
\hline & OR & IC (95\%) & Valor $p$ & OR & IC (95\%) & Valor $p$ \\
\hline \multicolumn{7}{|l|}{ Características pessoais } \\
\hline \multicolumn{7}{|l|}{ Sexo } \\
\hline Feminino & & & & 1 & & \\
\hline Masculino & & & & 0,50 & 0,$31 ; 0,81$ & 0,005 \\
\hline \multicolumn{7}{|l|}{ Uso de medicamento } \\
\hline Não & 1 & & & & & \\
\hline Sim & 2,23 & 1,$30 ; 3,82$ & 0,003 & & & \\
\hline \multicolumn{7}{|c|}{ Condições subjetivas de saúde bucal } \\
\hline \multicolumn{7}{|c|}{ Percepção da saúde bucal } \\
\hline Ótima/boa & & & & 1 & & \\
\hline Regular & & & & 1,81 & 1,$02 ; 3,20$ & 0,041 \\
\hline Ruim/péssima & & & & 2,69 & 1,$02 ; 7,11$ & 0,046 \\
\hline \multicolumn{7}{|c|}{ Percepção da aparência dentes e gengivas } \\
\hline Ótima/boa & 1 & & & & & \\
\hline Regular & 0,71 & 0,$36 ; 1,39$ & 0,321 & & & \\
\hline Ruim/péssima & 3,18 & 1,$56 ; 6,48$ & 0,001 & & & \\
\hline \multicolumn{7}{|c|}{ Comportamentos em saúde } \\
\hline \multicolumn{7}{|c|}{ Hábitos etilistas } \\
\hline Ausente & 1 & & & & & \\
\hline Presente & 0,61 & 0,$38 ; 0,96$ & 0,035 & & & \\
\hline \multicolumn{7}{|l|}{ Incapacidade funcional } \\
\hline \multicolumn{7}{|c|}{ Atividades instrumentais de vida diária } \\
\hline Independência & 1 & & & 1 & & \\
\hline Dependência parcial & 0,83 & 0,$35 ; 1,97$ & 0,675 & 2,18 & 0,$71 ; 6,72$ & 0,176 \\
\hline \multirow[t]{3}{*}{ Dependência total } & 2,551 & 1,$00 ; 6,27$ & 0,049 & 7,34 & 2,$29 ; 23,46$ & 0,001 \\
\hline & \multicolumn{3}{|c|}{ Domínio Social } & & & \\
\hline & OR & IC (95\%) & Valor $p$ & & & \\
\hline \multicolumn{7}{|c|}{ Condições subjetivas de saúde bucal } \\
\hline \multicolumn{7}{|c|}{ Percepção da aparência dentes e gengivas } \\
\hline Ótima/boa & 1 & & & & & \\
\hline Regular & 1,28 & 0,$68 ; 2,43$ & 0,439 & & & \\
\hline Ruim/péssima & 2,84 & 1,$34 ; 6,00$ & 0,006 & & & \\
\hline \multicolumn{7}{|c|}{ Incapacidade funcional } \\
\hline \multicolumn{7}{|c|}{ Atividades instrumentais de vida diária } \\
\hline Independência & 1 & & & & & \\
\hline Dependência parcial & 2,38 & 1,$02 ; 5,53$ & 0,044 & & & \\
\hline Dependência total & 2,22 & 0,$91 ; 5,44$ & 0,080 & & & \\
\hline
\end{tabular}

Domínio físico: Hosmer-Lemeshow - $X^{2}=1,33$; Valor de p=0,970; Domínio psicológico: Hosmer-Lemeshow - X²=3,96; Valor de p=0,555; Domínio social: Hosmer-Lemeshow $\mathrm{X}^{2}=0,480$; Valor de $\mathrm{p}=0,923$. 
estatisticamente significante entre os idosos com dependência total e independentes quanto à qualidade de vida ruim. Esse resultado pode representar o impacto percebido pelos idosos quando se dão contadas primeiras incapacidades funcionais que comprometem suas relações pessoais, o apoio recebido dos amigos e a atividade sexual. A incapacidade em funções como usar o telefone, fazer compras, manusear o dinheiro e realizar viagens podem ter como consequência um isolamento e distanciamento dos idosos de sua família e rede de amizades. Com o envelhecimento e aumento dos níveis de dependência, pode ocorrer um processo de adaptação, fazendo com que os idosos acostumem-se com uma nova situação de vida, representada por menor frequência de atividades sociais ${ }^{27,30}$.É comum em comunidades de cidades interioranas que se crie um pensamento de aceitação de condições debilitantes que afetem diversos indivíduos e estas condições acabam sendo aceitas como naturais ao processo de envelhecimento ${ }^{17,30}$.

Uma questão a ser levantada quanto às respostas no domínio social é a oportunização de atividades de lazer aos idosos. Este tipo de atividade deve ser encorajada e estimulada entre a população idosa, pois proporciona desenvolvimento das relações sociais, além de ser um importante fator de combate ao sedentarismo e motivador das funções cognitivas ${ }^{17}$. Sobre a relação quanto às Atividades Instrumentais de Vida Diária, pode-se afirmar que pode-se afirmar que quanto mais ativo e ocupado com atividades um idoso está, maior é seu prazer em relação a vida, havendo, portando, melhora em sua qualidade de vida ${ }^{15,23}$. É fundamental que as equipes de ESF promovam atividades em grupo, levando estas pessoas ao sentimento de integração e participação coletiva, condição que reduz potencialmente quadros depressivos e de desmotivação $0^{5,31}$.

Além da capacidade funcional, houve associação entre qualidade de vida geral ruim e sexo, uso de medicamentos, hábito etilista, percepção da saúde bucal e da aparência dos dentes e gengivas. O sexo masculino foi associado com menor chance de qualidade de vida ruim nos domínios físico e psicológico. Esse resultado pode ser explicado pelo fato das mulheres perceberem mais negativamente os efeitos do envelhecimento ${ }^{28,32,33}$. Em relação ao uso de medicamentos e reforçando o caráter subjetivo da autoavaliação da qualidade de vida, pode-se empreender o raciocínio de que a necessidade de uso contínuo de medicamentos venha imprimir no idoso uma consciência de uma menor condição de saúde ou até mesmo uma incapacidade subjetiva ${ }^{34}$.

No domínio físico, constatou-se menor chance de qualidade de vida ruim entre aqueles que possuíam hábitos etilistas; considera-se esta possibilidade a partir da característica de subjetividade desta análise, partindo da compreensão de que se trata de uma população moradora em cidade interiorana, com baixo nível de escolaridade e com características culturais que contribuem para mediadores sociais como álcool. Ainda, é possível refletir sobre prováveis efeitos do álcool na produção de ânimo e de segurança no enfrentamento dos problemas cotidianos.

A percepção negativa da saúde bucal e da aparência dos dentes e gengivas foi associada à maior chance de qualidade de vida geral ruim.
As condições de saúde bucal podem comprometer aspectos tais como fala, mastigação, deglutição, podem causar dor e desconforto ${ }^{17}$. Indivíduos que percebem sua saúde bucal como regular ruim ou péssima relatam um comprometimento na qualidade de vida nos seus domínios físico, social e psicológico. Esses resultados demonstram a importância da saúde bucal para o entendimento da qualidade de vida entre idosos, reafirmando sua natureza multifatorial e a necessidade de inclusão de medidas objetivas e subjetivas da saúde bucal quando o desfecho for qualidade de vida ${ }^{35}$. Vale ressaltar, que tanto as medidas de qualidade de vida e de percepção de saúde bucal adotadas nesse estudo são de natureza subjetiva, podendo ser superestimadas por uma visão mais negativa de vários aspectos da vida ${ }^{35,36}$.

Ainda que, durante a análise descritiva tenha sido possível observar idosos com condições negativas para o domínio ambiente, estas não apresentaram associações significativas quando confrontadas com as demais variáveis. Também é possível fomentar a discussão sobre a relação entre Atividades Instrumentais de Vida Diária e qualidade de vida no domínio ambiente para grupos de idosos em cidade interiorana; visto que, a observação de determinadas questões pode ser influenciada pelas facilidades de deslocamento e acesso humano à atenção a saúde.

Como estudo de base populacional que teve por característica a busca por indivíduos a partir de cadastros do Sistema Público de Saúde, ressalta-se que o número de participantes esteve na dependência da qualidade e atualização deste cadastramento. Vários aspectos que promovem a relação entre a qualidade de vida e a incapacidade funcional podem e devem ser trabalhados dentro do contexto de aproximação e acolhimento que norteia as ações da Atenção Primária à Saúde através da ESF.

Destaca-se como limitação do estudo a utilização de um instrumento genérico para avaliação da qualidade de vida dos idosos, uma vez que essa população possui características específicas que devem ser consideradas em qualquer avaliação. Entretanto, tantos os instrumentos de avaliação geral, como os de abordagem específica promovem importante influência nas decisões relacionadas à prevenção, intervenção e terapêutica das doenças, bem como na criação de estratégias e políticas de saúde ${ }^{37}$.

Os idosos pesquisados apresentam alta prevalência de qualidade de vida ruim em todos os domínios (físico, ambiente, social e psicológico). Quanto à avaliação das Atividades Instrumentais de Vida Diária, a maioria apresenta algum nível de dependência, por outro lado, grande parte dos idosos se mostra funcional para as Atividades Básicas de Vida Diária. A qualidade de vida ruim nos domínios físico, social e psicológico apresenta-se associada ao sexo masculino, uso de medicamentos, hábito etilista, percepção negativa da saúde bucal e da aparência dos dentes e gengivas e a idosos dependentes ou parcialmente dependentes.

\section{AGRADECIMENTOS}

Os autores agradecem a Prefeitura Municipal de Ibiaí-MG pelo apoio logístico. 


\section{REFERÊNCIAS}

1. Torres GV, Reis LA, Reis LA. Assessment of functional capacity in elderly residents of an outlying area in the hinterland of Bahia/ Northeast Brazil. Arq Neuropsiquiatr. 2010;68(1):39-42. http://dx.doi.org/10.1590/S0004-282X2010000100009

2. Del Duca GF, Silva MC, Hallal PC. Disability relating to basic and instrumental activities of daily living among elderly subjects. Rev Saúde Pública.2009;43(5):796-805. http://dx.doi.org/10.1590/S0034-89102009005000057

3. Santos RL, Virtuoso Júnior JS. Confiabilidade da versão brasileira da Escala de atividades instrumentais da vida diária. RBPS. 2008; 21(4):290-6.

4. Sposito G, D’Elboux MJ, Neri AL, Guariento ME. A satisfação com a vida e a funcionalidade em idosos atendidos em um ambulatório de geriatria. Ciênc Saúde Coletiva. 2013;18(12):3475-82. http://dx.doi.org/10.1590/S1413-81232013001200004

5. VitorinoLM, Paskulin LMG, Vianna LAC. Quality of life among older adults resident in long-stay care facilities. Rev Latino-Am Enfermagem. 2012;20(6):1186-95.

http://dx.doi.org/10.1590/S0104-11692012000600022

6. Somrongthong R, Wongchalee S, Ramakrishnan C, Hongthong $\mathrm{D}$, Yodmai K, Wongtongkam N. Influence of socioeconomic factors on daily life activities and Quality of Life of Thai Elderly. J Public Health Res. 2017;6(1):862.

http://dx.doi.org/10.4081/jphr.2017.862

7. Motta LB, Aguiar AC, Caldas CP. Estratégia Saúde da Família e a atenção ao idoso: experiências em três municípios brasileiros. Cad Saúde Pública. 2011;27(4):779-86. http://dx.doi.org/10.1590/S0102-311X2011000400017

8. Costa MFBNA, Ciosak SI. Atenção integral na saúde do idoso no Programa Saúde da família: visão dos profissionais de saúde. Rev Esc Enferm USP. 2010;44(2):437-44. http://dx.doi.org/10.1590/S0080-62342010000200028

9. Fleck MPA, Louzada S, Xavier M, Chachamovich E, Vieira G, Santos L, et al. Aplicação da versão em português do instrumento abreviado de avaliação da qualidade de vida "WHOQOL-bref". Rev Saúde Pública. 2000;34(2):178-83. http://dx.doi.org/10.1590/S0034-89102000000200012

10. Harper A, Power M. Steps for checking and cleaning data and computing domain scores for the WHOQOL-bref. Disponível em: http:// www.ufrgs.br/psiquiatria/psiq/sintaxe.pdf. Acesso em: 01 set. 2013.

11. World Health Organization (OMS). Quality os life Assessment (WHOQOL): position paper from the World Health Organization. Social Science and medicine. 1995; 41(10):403-9.

12. Barbosa BR, Almeida JM, Barbosa MR, Rossi-Barbosa LAR. Avaliação da capacidade funcional dos idosos e fatores associados à incapacidade. Ciênc Saúde Coletiva. 2014;19(8):3317-25. http://dx.doi.org/10.1590/1413-81232014198.06322013

13. Alves LC, Leite IC, Machado CJ. Conceituando e mensurando a incapacidade funcional da população idosa: uma revisão de literatura. Ciênc Saúde Coletiva. 2008; 13(4):1199-1207. http://dx.doi.org/10.1590/S1413-81232008000400016

14. Katz S, Ford AB, Moskowitz RW, Jackson BA, Jaffe MW. Studies of illness in the aged. The index of ADL: a standardized measure of biological and psychosocial function. JAMA. 1963;185(12):914-9. http://dx.doi.org/10.1001/jama.1963.03060120024016

15. Lino VTS, Pereira SEM, Camacho LAB, Ribeiro Filho ST, Buksman S. Adaptação transcultural da escala de independência em atividades da vida diária (Escala de Katz). Cad Saúde Pública 2008;24(1):103-12.

http://dx.doi.org/10.1590/S0102-311X2008000100010

16. Brasil. Ministério da Saúde. Departamento de Atenção Básica, Secretaria de Atenção à Saúde. Envelhecimento e saúde da pessoa idosa. Brasília: Ministério da Saúde; 2007.

17. Rubenstein LZ, Wieland D, English P, Josephson K, Sayre JA, Abrass IB. The Sepulveda VA Geriatric Evaluation Unit: data on four-year outcomes and predictors of improved patient outcomes. J Am Geriatr Soc. 1984;32(7):503-12. http://dx.doi.org/10.1111/j.1532-5415.1984.tb02235.x

18. Lenardt MH, Carneiro NHK, Binotto MA, Willig MH, Lourenco TM, Albino J. Fragilidade e qualidade de vida de idosos usuários da atenção básica de saúde. Rev Bras Enferm. 2016;69(3):478-83. http://dx.doi.org/10.1590/0034-7167.2016690309i

19. Rauchbach R, Wendling NM. Evolução da construção de um instrumento de avaliação do nível de atividade física para idosos Curitibativa. FIEP Bull. 2009;79(Esp):543-7.

20. Oliveira AC, Oliveira NMD, Arantes PMM, Alencar MA. Quality of life in elderly people who practice physical activity - a systematic review. Rev Bras Geriatr Gerontol. 2010;13(2):301-12. http://dx.doi.org/10.1590/S1809-98232010000200014

21. Locker D, Quinõnez C. To what extent do oral disorders compromise the quality of life? Community Dent Oral Epidemiol. 2011;39(1):3-11.

http://dx.doi.org/10.1111/j.1600-0528.2010.00597.x

22. Fuhrmann AC, Bierhals CCBK, Santos NO, Paskulin LMG. Associação entre a capacidade funcional de idosos dependentes e a sobrecarga do cuidador familiar. Rev Gaúcha Enferm. 2015;36(1):14-20.

http://dx.doi.org/10.1590/1983-1447.2015.01.49163

23. Buurman BM, Van Munster BC, Korevaar JC, Haan RJ, RooijSE.Variability in measuring (instrumental) activities of daily living functioning and functional decline in hospitalized older medical patients: a systematic review. J Clin Epidemiol. 2011; 64(6):619-27.

http://dx.doi.org/10.1016/j.jclinepi.2010.07.005

24. Brasil. Ministério da Saúde. Coordenação Nacional de Saúde Bucal. Projeto SB Brasil 2010: pesquisa nacional de saúde bucal: resultados principais. Brasília: Ministério da Saúde; 2011.

25. Lawton MP, Brody EM. Assessment of older people: selfmaintaining and instrumental activities of daily living. Gerontologist.1969;9(3):179-86. https://doi.org/10.1093/geront/9.3_Part_1.179

26. Freitas CV, Sarges ESNF, Moreira KECS, Carneiro SR. Avaliação de fragilidade, capacidade funcional e qualidade de vidados idosos atendidos no ambulatório de geriatria de um hospital universitário. Rev Bras Geriatr Gerontol. 2016;19(1): 119-28. http://dx.doi.org/10.1590/1809-9823.2016.14244

27. Cornejo M, Pérez G, de Lima KC, Casals-Peidro E, Borrell C. Oral health-related quality of life in institutionalized elderly in Barcelona (Spain). Med Oral Patol Oral Cir Bucal. 2013;18(2):285-92. http://dx.doi.org/10.4317/medoral.18280

28. Vasconcelos LCA, Prado Júnior RR, Teles JBM, Mendes RF. Self-perceived oral health among elderly individuals in a medium-sized city in Northeast Brazil. Cad Saúde Pública. 2012;28(6):1101-10. http://dx.doi.org/10.1590/S0102-311X2012000600009 
29. Lima BM, Araújo FA, Scattolin FAA. Qualidade de vida e independência funcional de idosos frequentadores do clube do idoso do município de Sorocaba. ABCS Health Sci. 2016;41(3):168-75. http://dx.doi.org/10.7322/abcshs.v41i3.907

30. Duarte YAO, Andrade CL, Lebrão ML. O Índex de Katz na avaliação da funcionalidade dos idosos. Rev Esc Enferm USP. 2007;41(2):317-25.

http://dx.doi.org/10.1590/S0080-62342007000200021

31. Oliveira DAS, Nascimento Júnior JRA, Bertolini SMMG, Oliveira DV. Participação de idosas em grupos sociais: qualidade de vida e capacidade funcional. Rev Rene. 2016; 17(2):278-84. http://dx.doi.org/10.15253/2175-6783.2016000200016

32. César CC, Mambrini JVM, Ferreira FR, Lima-Costa MF. Capacidade funcional de idosos: análise das questões de mobilidade, atividades básicas e instrumentais da vida diária via Teoria de Resposta ao Item. Cad Saúde Pública. 2015;31(5):931-45. http://dx.doi.org/10.1590/0102-311X00093214

33. Camara FM, Gerez AG, Miranda MLJ, Velardi M. Capacidade funcional do idoso: formas de avaliação e tendências. Acta Fisiatr. 2008;15(4):249-56.
34. Paiva MHP, Pegorari MS, Nascimento JS, Santos AS. Fatores associados à qualidade de vida de idosos comunitários da macrorregião do Triângulo do Sul, Minas Gerais, Brasil. Ciênc Saúde Coletiva. 2016; 21(11):3347-56.

http://dx.doi.org/10.1590/1413-812320152111.14822015

35. Giacomin KC, Peixoto SV, Uchoa E, Lima-Costa MF.A populationbased study on factors associated with functional disability among older adults in the Great Metropolitan Belo Horizonte, Minas Gerais State, Brazil. Cad Saúde Pública. 2008; 24(6):1260-70. http://dx.doi.org/10.1590/S0102-311X2008000600007

36. Campos ACV, Ferreira EF, Vargas AMD. Determinantes do envelhecimento ativo segundo a qualidade de vida e gênero. Ciênc Saúde Coletiva. 2015; 20(7):2221-37.

http://dx.doi.org/10.1590/1413-81232015207.14072014

37. Coluci MZO, Alexandre NMC, Milani D. Construction of measurement instruments in the area of health. Ciênc Saúde Coletiva. 2015;20(3):925-36.

http://dx.doi.org/10.1590/1413-81232015203.04332013 